\title{
A theoretical analysis of an alternative CUSUM statistic called CUSUM-slope for detecting signals from background noise in a low signal-to-noise environment
}

David Tam

Address: Department of Biological Sciences, University of North Texas, Denton, TX 76203, USA

Email: David Tam - dtam@unt.edu

from Eighteenth Annual Computational Neuroscience Meeting: CNS*2009

Berlin, Germany. 18-23 July 2009

Published: 13 July 2009

BMC Neuroscience 2009, I0(SuppI I):P83 doi:I0.I I86/I47I-2202-I0-SI-P83

This abstract is available from: http://www.biomedcentral.com/I47I-2202/I0/SI/P83

(c) 2009 Tam; licensee BioMed Central Ltd.

Signal detection in a low signal-to-noise environment, such as signals recorded from fMRI or EEG, is often a daunting task. In this paper, we limit the definition of noise to events that are generated from a random process whereas signal is defined as events that are generated from a non-random process. Numerous statistics were used to detect these non-random signals that are "buried" in a noisy background. One of these statistical techniques is the CUSUM method in which the cumulative sum of the data sequence (in most cases, a time-series of data) is used as the statistical variable for detecting significant changes (deviations) from the noise level. Most traditional CUSUM statistical measures rely on the cumbersome CUSUM-chart method. Here, a horizontal V-mask is used to provide an envelope in which if the CUSUM curve crosses this V-mask as the mask slides along the CUSUMcurve, it sets an "alarm" as detection and resets the origin of the CUSUM curve. Thus the analysis is origin-dependent and time-dependent.

The present paper introduces an alternate method, called CUSUM-slope [1], in which the "slope" of the CUSUMcurve is used as an approximation of the discrete-timederivative of the CUSUM curve to provide time-independence and origin-independence of the analysis. Furthermore, a theoretical proof is provided to show the CUSUM-slope method essentially captures the movingaverage of the signal with the background noise removed (filtered) in the time-window within which the CUSUM- slope is computed. Thus, the CUSUM-slope provides a simple detection method for not only extracting signal from noise, but also estimating signal duration and the times of signal onset (begin) and offset (end). A two-pass iterative procedure is also given to estimate the optimal time-window used in the CUSUM-slope analysis. The time-window variable can then be used as a criterion for detecting the stationarity of the signal (local vs. global stationarity) and the duration (extent) in which the signal may cease to exist during the detection period captured by the time-window length $t$.

\section{References}

I. Tam D: A theoretical analysis of cumulative sum slope (CUSUM-slope) statistic for detecting signal onset (begin) and offset (end) trends from background noise level. The Open Statistics \& Probability Journal 2009 in press. 